\title{
Aspectos psicosociales relacionados con la calidad de vida en mujeres con cáncer de mama
}

\section{Psychosocial aspects related to quality of life in women with breast cancer}

\author{
Fresia Hernández Moreno ${ }^{1}$ René Landero Hernández \\ Universidad Autónoma de Nuevo León, México
}

(Rec: marzo 2013 - Acep: mayo 2013)

\begin{abstract}
Resumen
El presente estudio tuvo como objetivo analizar la relación de las variables estrés, pensamientos negativos y apoyo social con la calidad de vida en mujeres con cáncer de mama. Se utilizó un muestreo no probabilístico de 50 mujeres diagnosticadas con cáncer de mama y un diseño transversal. Los resultados principales encontrados fueron las correlaciones negativas entre: estrés y calidad de vida; pensamientos negativos y apoyo social; pensamientos negativos y calidad de vida. También se encontró relación positiva entre las variables estrés y pensamientos negativos, y entre el apoyo social y la calidad de vida.
\end{abstract}

Palabras clave: cáncer de mama, calidad de vida, estrés, pensamientos negativos, apoyo social.

\begin{abstract}
The present study aimed to analyze the relationship of the variables stress, negative thoughts and social support to the quality of life in women with breast cancer. We used a non-probability sample of 50 women diagnosed with breast cancer and a cross-sectional design. The main results were found negative correlations between: stress and quality of life, social support and negative thoughts, negative thoughts and quality of life. We also found a positive relationship between the variables stress and negative thoughts and finally, positive relationship between social support and quality of life.
\end{abstract}

Keywords: breast cancer, quality of life, stress, negative thoughts, social support.

\footnotetext{
1 Correspondencia a: Fresia Hernández Moreno. Facultad de Psicología, Universidad Autónoma de Nuevo León, México. Dirección postal: c/Dr. Carlos Canseco (Esquina con E. Aguirre Pequeño N 110. Col. Mitras Centro. C. P. 64460. Monterrey, NL., México. Tel: (81) 8348 38 66. E-mail: fresiahdez@ hotmail.com.
} 


\section{Introducción}

Durante la última década, el cáncer se ha convertido en una de las principales causas de mortalidad a nivel mundial. Del cual, el cáncer de mama ocupa el primer lugar de defunciones por esta enfermedad en población de mujeres (Organización Mundial de la Salud [OMS], 2012).

En México, una tercera parte de las mujeres que se diagnostican con cáncer mamario mueren debido a esta enfermedad, lo que equivale a doce mujeres cada día (Knaul, López-Carrillo, Lazcano, Gómez, Romieu $\&$ Torres, 2009).

Desde el año 2006 el cáncer de mama se ha convertido en la primera causa de mortalidad en mujeres de 30 a 54 años y la primera por cáncer, desplazando al cáncer cérvico uterino que anteriormente era el que estaba en primer lugar de muertes por este padecimiento (Knaul, Nigenda, Arreola-Ornelas, Langer \& Frenk, 2009). Es por esto que el cáncer de mama es desde hace algunos años, un tema de gran importancia para los profesionales de la salud mental, por las consecuencias complejas que tiene en diversas áreas de su vida, ya sea en lo psicológico, social, familiar, además de los problemas económicos que pueden surgir debido a los costos derivados de esta enfermedad (Vinaccia \& Orozco, 2005).

En cuanto a las variables psicológicas, existe evidencia de que están asociadas al cáncer, ya sea como factores detonantes o facilitadores de su aparición, así como durante el desarrollo de la enfermedad o posible recaída (Vera-Villarroel \& Buela-Casal, 1999).

La calidad de vida está relacionada de manera importante con la salud, haciendo referencia a las valoraciones que tiene el individuo en cuanto a la percepción de salud, tanto en aspectos subjetivos como objetivos (Ramírez, 2007), en particular en las mujeres con cáncer de mama, ya que este padecimiento provoca alteraciones importantes en diversas áreas de las pacientes, además de que está relacionada con la adaptación a la enfermedad y la respuesta a los tratamientos (Moreno $\&$ Kern, 2005). Por lo anterior, se ha hecho importante evaluar y conocer el impacto de la enfermedad y tratamiento sobre la percepción de bienestar del paciente (Martín, Sánchez \& Sierra, 2005) esta valoración debe de ser integral, considerando el aspecto físico, social y psicológico (Montes, Mullins \& Urrutia, 2006).

El padecer cáncer de mama, es un acontecimiento estresante que amenaza de manera significativa a las personas en distintas áreas (Fawzy \& Greenberg, 1996) y puede presentar decadencia de las actividades diarias, la pérdida del sentido de vida y provocar estados emocionales negativos (GilRoales, 2004). En general las enfermedades crónicas suelen afectar de manera importante en diversos aspectos de la vida de los pacientes (Oblitas, 2006). Eventos como padecer una enfermedad crónica, pueden ser capaces de originar emociones y de activar procesos de pensamientos acerca de los cuales las personas tienden a perturbarse a sí mismas (Ellis, 1987). Estos pensamientos pueden convertirse en distorsiones cognitivas, las cuales son interpretaciones exageradas e incongruentes de la realidad (Beck, Rush, Shaw \& Emery, 2000),y pueden ser usadas como el medio en que el individuo percibe y construye su mundo (Calvete \& Connor-Smith, 2005).

Los estados emocionales influyen en la salud de manera directa, en el funcionamiento fisiológico, el reconocimiento de síntomas, la búsqueda de atención médica oportuna, así como también en la realización de conductas saludables y no saludables y en la percepción y recepción de apoyo social. Cuando una persona está bajo un estado de ánimo negativo, se convierte más vulnerable a enfermedades infecciosas, se eleva la percepción y reporte de síntomas y se reduce la búsqueda de atención médica oportuna. A partir de lo anterior, se puede explicar cómo el pesimismo y el ánimo depresivo, se pueden asociar con un mayor efecto negativo del estrés sobre la salud (Barra, Cerna, Kramm \& Vélez, 2004).

En los últimos años se ha incrementado el interés por estudiar cómo las personas afrontan las situaciones difíciles y estresantes (Chico, 2002); aumentando la atención en los recursos psicológicos que mejoran la salud, ya que estos, como factores protectores relacionados con la salud y el bienestar, aumentan la resistencia a la adversidad (Remor, Amorós \& Carrobles, 2006). En este sentido, el apoyo social funciona como factor protector en el enfermo oncológico permitiendo disminuir la intensidad de los efectos y los cambios asociados a la enfermedad (Cano, 2005), así como también, permitiendo que les sea más fácil adaptarse a los cambios y enfrentar las crisis que se presenten, protegiendo al individuo de estados patológicos. También contar con redes de apoyo social puede ayudar reducir la cantidad de medicamentos utilizados en el tratamiento, además de acelerar la recuperación y facilitar la adherencia al tratamiento (Cobb, 1976).

Es por lo anterior que el presente trabajo tuvo como objetivo analizar la relación del estrés, pensamientos negativos y apoyo social, con la calidad de vida en mujeres con cáncer de mama. 


\section{Método}

\section{Participantes}

Se llevó a cabo la recolección de los datos con mujeres diagnosticadas con cáncer de mama, acudiendo al Hospital de Gineco Pediatría No. 2, del Instituto Mexicano del Seguro Social, en la ciudad de Los Mochis, México, con previa autorización para la aplicación de los instrumentos. Se logró recolectar una muestra de 50 mujeres diagnosticadas entre 34 y 73 años de edad, con una media de 48.7 (D.E=9.41), con un promedio de tiempo de diagnóstico de 17.3 meses (D.E $=18.6$ ); las cuales fueron entrevistadas en el Hospital de Ginecología y Obstetricia del IMSS en la ciudad de Los Mochis, Sinaloa.

\section{Procedimiento}

Se llevó a cabo la recolección de los datos acudiendo al hospital, con previa autorización de las autoridades correspondientes para la aplicación de los instrumentos; estos fueron auto aplicables para cada una de las pacientes, primeramente firmando el consentimiento informado de participación voluntaria en la investigación y respetando la confidencialidad de quienes contestaron.

Después de recolectados los datos, se procedió a la captura de la información obtenida en el programa estadístico SPSS 16, calculando primeramente la consistencia interna de cada una de las escalas a través del alfa de Cronbach, así como los estadísticos descriptivos de cada variable.

Posteriormente se analizaron las correlaciones entre las variables, utilizando el coeficiente de Spearman, ya que las escalas no se distribuían con normalidad de acuerdo a los resultados de la prueba KolmogorovSmirnov $(\mathrm{p}<.05)$.

\section{Instrumentos}

Para medir el estrés se utilizó la Escala de Estrés Percibido (Perceived Stress Scale, PSS) elaborada por Cohen, Kamarak y Mermelstein (1983) para evaluar el grado en que las situaciones de la vida son valoradas como estresantes, obteniendo un alfa de Cronbach de .75. En este estudio se utilizó la adaptación cultural realizada en México por González y Landero (2007), la cual cuenta con 14 ítems y una consistencia interna $(\mathrm{a}=.83)$, además de confirmar la estructura factorial de la escala original, utilizando un análisis factorial confirmatorio. La puntuación va de nunca -0- a muy a menudo -4-. Invirtiéndose la puntuación en los ítems: $4,5,6,7,9,10$ y 13 . La escala cuenta con un rango de puntuaciones posibles que van de 0 a 56 . La mayor puntuación corresponde a mayor estrés percibido.

Para la medición de la calidad de vida se utilizó el instrumento QLQ-C30 3.0 que fue elaborado por el grupo de Calidad de Vida de la Organización Europea para la Investigación y Tratamiento del Cáncer (EORTC) la cual cuenta con una confiabilidad de entre $0.70 \mathrm{y}$ 0.87 (Fayers, Aaronson, Bjordal, Groenvold, Curran, $\&$ Bottomley, 2001). Incluye cinco escalas funcionales: funcionamiento físico, rol emocional, social y cognitivo; tres escalas de síntomas: fatiga, dolor, y náusea/vómitos y una escala global de salud/calidad de vida. Además de ítems individuales que evalúan síntomas adicionales de disnea, pérdida de apetito, insomnio, estreñimiento y diarrea, y el impacto financiero. Consta de 30 preguntas las cuales cuentan con un formato de respuesta tipo Likert de cuatro puntos, excepto en la escala global que tiene siete. Todas las escalas e ítems individuales se convierten en una puntuación de 0 a 100, siendo este el rango de puntuación posible para la escala.

\section{Apoyo social}

Para la evaluación del apoyo social se utilizó el cuestionario de apoyo social percibido (MOS) de Sherbourne y Stewart (1991), el cual cuenta con 20 ítems. El ítem número 1 hace referencia al tamaño de la red social y los 19 ítems restantes están referidos a cuatro dimensiones del apoyo social funcional: emocional/informacional, instrumental, interacción social positiva y apoyo afectivo. Las opciones de respuesta están dadas a través de una escala likert de 1 (Nunca) a 5 (Siempre). En este estudio se utilizó la versión abreviada de Gjesfjeld, Greeno y Kim (2008), de 12 ítems y con una consistencia interna de .94 según el alfa de Cronbach. Cuestionario de Pensamientos Automáticos (ATQ-R)

Revisado (Kendall \& Hollon, 1989). El ATQ-R es una medida cognitiva de frecuencia de pensamientos automáticos asociados con el ánimo depresivo. Cada ítem se evalúa en una escala de 5 puntos $(1=$ no en absoluto, nunca, $2=$ alguna vez, $3=$ bastantes veces, $4=$ muchas veces, $5=$ todo el tiempo). La adaptación del ATQ-R al castellano fue realizada a través del método de traducción-retrotraducción (Calvete \& Connor-Smith, 2005), quienes agruparon los ítems en cuatro subgrupos mediante un sistema de jueces. El 
coeficiente alfa de Cronbach fue de .94 para la adaptación al castellano.

\section{Resultados}

En los resultados presentados enseguida, primero se analizaron los estadísticos descriptivos de las variables sociodemográficas y de las variables psicosociales, así como, la consistencia interna de cada una de las escalas a través del alfa de Cronbach.

Se puede observar en la Tabla 1 que la edad promedio fue de 48.72 (D.E = 9.41), con una escolaridad promedio de 9.81 años $(\mathrm{D} . \mathrm{E}=4.09)$.

Tabla 1.

Variables Sociodemográficas $(n=50)$

\begin{tabular}{lccc}
\hline & Mediana & Media & D.E. \\
\hline Edad & 48.5 & 48.72 & 9.41 \\
Escolaridad & 9.00 & 9.81 & 4.09 \\
\hline
\end{tabular}

La consistencia interna de las escalas de acuerdo con el coeficiente alfa de Cronbach, es adecuada para cada una de ellas. Las puntuaciones se muestran en la Tabla 2.

Tabla 2.

Consistencia interna de las escalas

\begin{tabular}{lc}
\hline Escala & Alfa \\
\hline Escala de Apoyo Social & .87 \\
Pensamientos negativos (ATQ) & .92 \\
Calidad de Vida & .88 \\
Estrés & .64 \\
\hline
\end{tabular}

Respecto a las variables psicosociales tomadas en cuenta para este estudio, se obtuvo un promedio de apoyo social de 44.5 (D.E. $=9.41$ ). En pensamientos negativos la media fue de 31.3 (D.E. = 19.7). En cuanto a la calidad de vida la puntuación media fue de 72.3 (D.E. $=13.8$ ). El promedio de estrés fue 24.5 (D.E. = 6.5) (Tabla 3).

Tabla 3.

Estadísticos descriptivos de las variables $(n=50)$

\begin{tabular}{lccc}
\hline Escala & Mediana & Media & D.E \\
\hline Apoyo Social & 46 & 44.5 & 9.41 \\
ATQ & 30.5 & 31.26 & 19.69 \\
Calidad de vida & 74.22 & 72.27 & 13.82 \\
Estrés & 25 & 24.48 & 6.48 \\
\hline
\end{tabular}

Se realizó el análisis de correlación entre las variables, y se obtuvieron las siguientes correlaciones significativas: estrés y calidad de vida $\left(\mathrm{r}_{\mathrm{s}}=-.394, \mathrm{p}=\right.$ $.005)$, estrés y pensamientos negativos $\left(\mathrm{r}_{\mathrm{s}}=.639, \mathrm{p}=\right.$ $.001)$, pensamientos negativos y apoyo social $\left(r_{s}=-.502\right.$, $\mathrm{p}=.001)$, calidad de vida y pensamientos negativos $\left(\mathrm{r}_{\mathrm{s}}\right.$ $=-.537, \mathrm{p}=.001)$ y calidad de vida con apoyo social $\left(\mathrm{r}_{\mathrm{s}}=.327, \mathrm{p}=.021\right)$.

\section{Discusión}

De acuerdo con los objetivos de esta investigación, se encontró que existe una relación entre los pensamientos negativos y el estrés, ya que el hecho de padecer una enfermedad crónica puede originar emociones y activar procesos de pensamientos los cuales tienden a perturbar a las personas (Ellis, 1987), además el hecho de enfrentarse a una situación de este tipo, se puede interpretar como peligrosa, debido a las consecuencias severas que puede tener esta enfermedad sintiéndose incapaz de afrontarla (Beck et al., 1983; Ellis \& Grieger, 1990).

Lo anterior, se puede explicar según el modelo transaccional del estrés, en el que los procesos cognitivos y emocionales influyen de acuerdo a cómo se enfrenta y maneja un evento (Lázarus \& Lázarus, 1994). Por lo tanto, si una persona evalúa el hecho de tener cáncer de manera negativa y experimenta la presencia de pensamientos negativos determinará que esta situación resulte estresante. Al contrario de esto Gaviria, Vinaccia, Riveros y Quiceno (2007) encontraron en su estudio de pacientes con cáncer en tratamiento quimioterapéutico frecuentes manifestaciones de evitación a nivel conductual y emocional expresando poco sus sentimientos, mientras que a nivel cognitivo presentaron supresión de pensamientos negativos sustituyéndolos por pensamientos positivos que les favorecieran tolerar la enfermedad.

La presencia de este tipo de distorsiones cognitivas, está además relacionada con la percepción que esta tenga del apoyo social, ya que se ha encontrado una relación negativa pues, a mayor pensamientos negativos, menor apoyo social percibido. Coincidiendo con los resultados de Villa y Vinaccia (2009). Por el contrario, Font y Cardoso (2009), han encontrado que a nivel de pensamientos, predomina el pensar que la enfermedad se debe superar y no se debe intentar buscarle sentido.

También, se encontró la relación negativa entre el estrés y la calidad de vida, esto concordando con un estudio de 97 pacientes con probabilidad de cáncer de mama, de las cuales 73 de ellas fueron diagnosticadas, 
se encontró en este grupo que tenían peor calidad de vida física y psicológica, así como mayor nivel de estrés y problemas de salud, en comparación a las que presentaban únicamente algún tipo de tumor benigno en la mama. Además de que los problemas de salud y el estrés fueron los predictores más importantes en relación a la calidad de vida en este grupo de pacientes (Ju-Yu et al., 2006). Como ya se ha mencionado anteriormente, el padecimiento de cáncer de mama, supone una serie de cambios y situaciones estresantes en distintos niveles de la persona, ya que cambia drásticamente el estilo de vida de ella y de quienes están a su alrededor, además de que ésta se ve como una enfermedad muy amenazante por el temor a la muerte y a los tratamientos médicos a los que se enfrentan.

Asimismo, esto está asociado a la relación que existe entre el apoyo social y el estrés, el cual nos indica que a menor apoyo social, mayor es el estrés, lo cual nos dice que funciona de manera importante como amortiguador y protector de la aparición del estrés y pensamientos negativos. Estos resultados son consistentes con los encontrados por Castro, Campero y Hernández (1997), Cohen y Wills (1985), Juárez y Landero (2009), Turner-Cobb, Sephton, Koopman, Blake-Mortimer y Spiegel (2000). También, estos resultados coinciden con los de Del Mar González et al. (2001), que comprobaron que un uso más frecuente de estrategias de búsqueda de apoyo social, se acompañaba de menor malestar emocional, funcionando como factor protector contra el estrés que provoca una enfermedad como lo es el cáncer de mama.

La presencia de pensamientos negativos se ha asociado a una menor calidad de vida percibida en este tipo de pacientes, como lo confirma este estudio mostrando una correlación significativa entre dichas variables. Esto se puede explicar desde la influencia de esta variable en la aparición y mantenimiento del estrés.

En un estudio realizado por Vinaccia et al. (2005) en pacientes con cáncer pulmonar, se encontraron puntajes de calidad de vida adecuados, explicados por la manifestación de apoyo social del personal de salud y amigos, ya que el apoyo por parte de la familia, aunque era más frecuente, lo percibían como pobre. Estos resultados apoyan los encontrados en nuestra investigación, donde a mayor apoyo social, mejor será la calidad de vida de las pacientes.

Retomando lo anterior, se sugiere que en futuros estudios se implementen estrategias de tipo longitudinal que permitan detectar los momentos en los que se presentan estas variables psicosociales relacionadas con la calidad de vida.
Otra recomendación sería trabajar de manera conjunta con el equipo médico del hospital donde se realice alguna investigación similar, ya que esto facilitaría de manera importante la obtención de información relevante de las pacientes, como el acceso al historial médico, esto con el fin de relacionar variables psicológicas y aspectos médicos de la enfermedad.

En base a lo mencionado anteriormente, se sugiere realizar intervenciones psicológicas que se basen en los resultados de las investigaciones, siendo oportunos en las necesidades específicas que tienen las pacientes al momento de enfrentar la enfermedad y el tratamiento del cáncer, en este caso, trabajar en el manejo del estrés y reducción de los pensamientos negativos.

\section{Referencias}

Barra, E., Cerna, R., Kramm, D. \& Vélez, V. (2004). Problemas de salud, estrés, afrontamiento, depresión y apoyo social en adolescentes. Terapia Psicológica, 24(1), 55-61. Recuperado de http:// www.redalyc.org/articulo.oa?id=78524106

Beck, A., Rush, A. \& Shaw, B. (2000). Terapia cognitiva de la depresión (2a ed.). España: Desclée.

Beck, A. T. (1983). Cognitive therapy of depression: new perspectives. En P. J. Clayton \& J. E. Barrett (Eds.). Treatment of Depression: Old Controversies and New Approaches (265-289). New York: Raven Press.

Calvete, E. \& Connor-Smith, J. (2005). Automatic thoughts and psychological symptoms: A Cross-Cultural Comparison of American and Spanish students. Cognitive Therapy and Research, 29(2), 201-207. doi:10.1007/s10608-005-3165-2

Cano, A. (2005). Control emocional, estilo represivo de afrontamiento y cáncer: ansiedad y cáncer. Psicooncología, 2(1), 71-80. Recuperado de http://revistas.ucm.es/index.php/PSIC/article/view/ PSIC0505120071A/16099

Cardona, P. G., Nicholson, B. C. \& Fox, R. A. (2000). Parenting among Hispanic and Anglo- American mothers with young children. The Journal of Social Psychology, 140(3), 357-365. doi:10.1080/00224540009600476

Castro, R., Campero, L. \& Hernández, B. (1997). La investigación sobre apoyo social en salud: situación actual y nuevos desafíos. Journal of public health, 31(4), 425-435. doi:10.1590/S003489101997000400012

Chico, E. (2002). Optimismo disposicional como predictor de estrategias de afrontamiento. Psicothema, 14(3), 544-550. Recuperado de http://www.psicothema.com/pdf/3177.pdf

Cobb, S. (1976). Social support as a moderator of life stress. Psychosomatic Medicine, 38(5), 300-314.

Cohen, S. \& Wills, T. (1985). Stress, social support, and the buffering hypothesis. Psychological Bulletin, 98(2), 310-357. doi:10.1037/0033-2909.98.2.310

Cohen, S., Kamarck, T. \& Mermelstein, R. (1983). A global measure of perceived stress. Journal of Health and Social Behavior, 24(4), 385-396. Recuperado de http://www.psy.cmu.edu/ scohen/ globalmeas83.pdf

Del Mar González-Tablas, M., Palenzuela, D., Palido, R., Saez, L. \& López, E. (2001). The role of generalized expectancies of control on coping and psychological adjustment in women with breast cáncer. Ansiedad y Estrés, 7(1), 1-14. 
Ellis, A. \& Grieger, R. (1990). Manual de terapia racional-emotiva (volumen 2). Bilbao: Descleé.

Ellis, A. (1987). The practice of rational-emotive therapy (2a ed.) Nueva York: Springer Pub Co.

Espínola-Rodríguez, S. \& Carmelo, E. (2010). Validación Argentina del cuestionario MOS de Apoyo Social Percibido. Psicodebate, 7, 155-168. Recuperado de http://www.palermo.edu/cienciassociales/publicaciones/pdf/Psico7/7Psico\%2010.pdf

Fawzy, F. \& Greenberg, D. (1996). Oncology. En J. Rundel \& M.Wise, Textbook of consultation-Liarson Psychiatric (pp. 155168). Washington: The American Psychiatric Press.

Fayers, P., Aaronson, N., Bjordal, K., Groenvold, M., Curran, D. \& Bottomley, A. (2001). The EORTC QLQ-C30 Scoring Manual (3a ed.). Bruselas: European Organization for Reserch and Treatment of Cancer.

Font, A. \& Cardoso, A. (2009). Afrontamiento en cáncer de mama: pensamientos, conductas y reacciones emocionales. Psicooncología, 6(1), 27-42. Recuperado de http://revistas.ucm.es/index.php/ PSIC/article/view/PSIC0909120027A

García-Viniegras, C. \& González, M. (2007). Bienestar psicológico y cáncer de mama. Avances en psicología latinoamericana, 25(1), 72-80. Recuperado de http://www.redalyc.org/articulo. oa? id=79902508

Gaviria, A., Vinaccia, S., Riveros, M. \& Quiceno, J. (2007). Calidad de vida relacionada con la salud: afrontamiento del estrés y emociones negativas en pacientes con cáncer en tratamiento quimioterapéutico. Psicología desde el Caribe, 20, 50-75. Recuperado de http://ciruelo.uninorte.edu.co/pdf/psicologia_caribe/20/3_Calidad\%20de\%20vida.pdf

Gil- Roales, J. (2004). Psicología de la salud: Aproximación histórica, conceptual y aplicaciones. Madrid: Pirámide.

Gjesfjeld, Ch., Greeno, G. \& Kim, K. (2008). A Confirmatory Factor Analysis of an Abbreviated Social Support Instrument: The MOS-SSS. Research on Social Work Practice, 18(3), 231-237. doi: $10.1177 / 1049731507309830$

González, M. \& Landero, R. (2007). Factor Structure of the Perceived Stress Scale (PSS) in a Sample from Mexico. The Spanish Journal of Psychology, 10(1), 199-206. Recuperado de http://www.psy. cmu.edu/ scohen/Factor\%20Structure_Mexico.pdf

Juárez, D. \& Landero, R. (2009). Variables psicosociales y salud en mujeres con cáncer de mama. SUMMA Psicológica UST, 6(2), 79-88. Recuperado de http://dx.doi.org/10.18774/summa-vol6. num2-64

Ju-Yu, Y., Chih-Hung, K., Cheng-Fang, Y., Ming-Jen, Y., Chiou-Yuh, W., Chiung-Hui, J., \& Ming-Feng, H. (2006). Quality of life, depression, and stress in breast cancer women outpatients receiving active therapy in Taiwan. Psychiatry \& Clinical Neurosciences, 60(2), 147-153. doi:10.1111/j.1440-1819.2006.01479.x

Kendall, P. \& Hollon, S. (1989). Anxious self-talk: Development of the Anxious Self-Statements Questionnaire (ASSQ). Cognitive Therapy and Research, 13(1), 81-93. doi:10.1007/BF01178491

Knaul, F., López-Carrillo, L., Lazcano-Ponce, E., Gómez, H., Romieu, I. \& Torres, G. (2009). Cáncer de mama: un reto para la sociedad y los sistemas de salud. Salud Pública de México, 51(2), 135-137. Recuperado de http://bvs.insp.mx/rsp/articulos/ articulo_e2.php?id=002285

Knaul, F., Nigenda, G., Lozano, R., Arreola-Ornelas, H., Langer, A. \& Frenk, J. (2009). Cáncer de mama en México: una prioridad apremiante. Salud Pública de México, 51(2), 335-344. Recuperado de http://bvs.insp.mx/rsp/articulos/articulo_e4.php?id=002305
Lazarus, R. S. \& Lazarus, B. N. (1994). Pasión y razón: la comprensión de nuestras emociones. Barcelona: Editorial Paidós.

Martín, J., Sánchez, M. \& Sierra, J. (2005). Evaluación de la Calidad de vida en pacientes con cáncer: una revisión. Revista Colombiana de Psicología, 14, 34-45. Recuperado de http://www.redalyc.org/ pdf/804/80401403.pdf

Montes, L., Mullins, M. \& Urrutia, M. (2006). Calidad de vida en mujeres con cáncer cérvico uterino. Revista chilena de ginecología y obstetricia, 71(2), 129-134. doi:10.4067/S071775262006000200010

Moreno, B. \& Kern, E. (2005). Calidad de vida relacionada con la salud infantil y el trasplante de órganos: una revisión de literatura. Revista Colombiana de Psicología, 14, 46-52. Recuperado de http://www.redalyc.org/pdf/804/80401404.pdf

Oblitas, L. (2006). Psicología de la Salud y Calidad de Vida. México: Thompson.

Organización Mundial de la Salud (Febrero, 2012). Cáncer. (OMS Publicación No 297). Recuperado de http://www.who.int/mediacentre/factsheets/fs297/es/

Ramírez, F. (2007). Calidad de vida relacionada con la salud como medida de resultados en salud: revisión sistemática de la literatura. Revista Colombiana de Cardiología, 14(4), 207-222. Recuperado de http://www.scielo.org.co/pdf/rcca/v14n4/v14n4a4.pdf

Remor, E., Amorós, M. \& Carrobles, J. (2006). Optimismo y la experiencia de la ira en relación con el malestar físico. Anales de psicología, 22(1), 37- 44. Recuperado de http://www.um.es/ analesps/v22/v22_1/05-22_1.pdf

Schwartzmann, L. (2003). Calidad de vida relacionada con la salud: aspectos conceptuales. Ciencia y Enfermería, 9(2), 9-21. doi: 10.4067/S0717-95532003000200002

Secretaría de Salubridad y Asistencia (2008). Rendición de cuentas en salud 2008. Recuperado de www.dged.salud.gob.mx/contenidos/ dedss/descargas/rcs/rcs_2008.pdf

Sherbourne, C. D. \& Stewart, A. L. (1991). The MOS Social Support Survey. Social Science \& Medicine, 32(6), 705-714. doi:10.1016/0277-9536(91)90150-B

Turner-Cobb, J., Sephton, S., Koopman, C., Blake-Mortimer, J. \& Spiegel, D. (2000). Social Support and Salivary Cortisol in Women With Metastatic Breast Cancer. Psychosomatic Medicine, 62(3), 337-345. doi:10.1097/00006842-200005000-00007

Vera-Villarroel, P. \& Buela-Casal, G. (1999). Psiconeuroinmunología: Relaciones entre factores psicológicos e inmunitarios en humanos. Revista latinoamericana de Psicología, 31(2), 271-289. Recuperado de http://www.redalyc.org/pdf/805/80531204.pdf

Villa, I. \& Vinaccia, S. (2006). Adhesión terapéutica y variables psicológicas asociadas en pacientes con diagnóstico de VIH-sida. Psicología y Salud, 16(1), 51-62. Recuperado de http://revistas. uv.mx/index.php/psicysalud/article/view/792/1436

Vinaccia, S. \& Orozco, L. (2005). Aspectos psicosociales asociados con la calidad de vida de personas con enfermedades crónicas. Diversitas, 1(2), 125-137. Recuperado de http://www.redalyc.org/ articulo.oa? id $=67910202$

Vinaccia, S., Quiceno, J., Fernández, H., Contreras, F., Bedoya, M., Tobón, S. \& Zapata, M. (2005). Calidad de vida, personalidad resistente y apoyo social percibido en pacientes con diagnóstico de cáncer pulmonar. Psicología y Salud, 15(2), 207-220. Recuperado de http://revistas.uv.mx/index.php/psicysalud/article/ view/805/1459\#sthash.fr4yrxQ6.dpuf 